\title{
Johann Moritz Rugendas oder die Malerei auf Reisen
}

Anders als bei Ida Pfeiffer, bei Fredrika Bremer und selbst bei Flora Tristan, die in Frankreich ihre eigentliche Hölle erlebte, aber ein Paradies wie ihr Enkel Paul Gauguin nicht finden konnte, bereist Johann Moritz Rugendas eine Welt, die weder eine Hölle noch ein Paradies ist. Zusammen mit seinen Crayons, seinen Kohlestiften, Aquarellpinsel und Ölfarben führt der in Augsburg geborene Rugendas die Ideen Alexander von Humboldts im Gepäck mit sich, Vorstellungen, in denen - wie Humboldt dies in seinem Kosmos äußerte - der Landschaftsmalerei eine große, eine immense Bedeutung zukommt. Nicht nur die Feder, auch Stift und Pinsel gehen auf Reisen. Dies sei in einer Vorlesung über das Verhältnis von Reisen und Schreiben zumindest kurz vermerkt.

Der junge Rugendas bereist den amerikanischen Kontinent in gewisser Weise auf den Spuren des preußischen Gelehrten, auch wenn er vielfach von dessen Reiserouten abwich, hatte ihm Alexander von Humboldt doch eine Vielzahl von Wegen durch das tropische Amerika gewiesen. Vor allem aber reiste Johann Moritz Rugendas als Schüler Humboldts, auch wenn er sich nicht nur für das tropische Amerika, sondern auch für die Außertropen des amerikanischen Kontinents interessierte. Mit seinem Pinsel entwarf er jene Landschaftsmalerei des 19. Jahrhunderts, wie sie Humboldt vorschwebte.

Ein kurzer biographischer Abriss des Lebens des Augsburger Reisemalers, der aus einer wahren Malerdynastie stammte, erübrigt sich an dieser Stelle, kann hier doch nicht mehr als ein kleiner Hinweis auf Rugendas und eine ganze Vielzahl von Reisemalern gegeben werden, die zum Teil mit ungeheurem Engagement die Neue Welt durchstreiften. Freilich können hier auch nur wenige Verweise auf den Verlauf der beiden Reisen von Rugendas durch den amerikanischen Kontinent gegeben werden, verdienen seine Figur wie seine Reisen doch eine weitaus intensivere Beschäftigung, die hier jedoch nicht zu leisten ist. ${ }^{1}$

Rugendas kam - wie bereits erwähnt - im Rahmen der russischen Expedition von Langsdorff erstmals nach Brasilien, und zwar im März 1822. Als Maler begleitete er diese Expedition dann von Mai bis November 1824 nach Minas Gerais; doch trennte er sich zu diesem Zeitpunkt dann endgültig vom Leiter der Expedition, mit dem er sich bereits bald nach der Ankunft in Brasilien während

\footnotetext{
1 Vgl. zu Johann Moritz Rugendas u. a. Diener, Pablo: Johann Moritz Rugendas. Bilder aus Mexico. Augsburg: Wissner Verlag 1993; ders.: Rugendas. América de punta a cabo. Rugendas y la Araucanía. Santiago de Chile: Editorial Aleda 1992; sowie ders.: Johann Moritz Rugendas 1802-1856. Augsburg: Wissner Verlag 1998.
}

¿ Open Access. () 2020 Ottmar Ette, publiziert von De Gruyter. (cc) BY-NC-ND Dieses Werk ist lizenziert unter der Creative Commons Attribution-NonCommericial-NoDerivatives 4.0 Lizenz.

https://doi.org/10.1515/9783110650686-025 
Abb. 83: Johann Moritz Rugendas (Augsburg, 1802 - Weilheim an der Teck in Württemberg, 1858).

der langen untätigen Wartezeit zerstritten hatte. Im Mai 1825 kehrte Rugendas wieder nach Europa zurück. ${ }^{2}$ Dies war ein Beispiel für das schwierige Zusammenleben von Künstlern und Wissenschaftlern, wie es sich in der Geschichte wissenschaftlicher Reisen vielfach zutrug. Martius und Spix etwa fanden für ihre Brasilien-Expedition überhaupt keine Künstler, die bereit waren, sich den Anforderungen der Wissenschaft zu unterwerfen.

Johann Moritz Rugendas' zweite, die sogenannte 'große' Amerikareise hatte ihren Auftakt in Mexico ab Juli 1831 mit der Ankunft im karibischen Hafen von Veracruz. Daran schlossen sich unterschiedliche Reisen innerhalb von Mexico an, von denen wir ein reiches Bildmaterial besitzen. Die Weiterreise erfolgte dann über die Pazifikküste mit der Ankunft in Chile im Juli 1834, wobei sich Rugendas zunächst im Hafen von Valparaíso aufhielt, um dann in Santiago de Chile dauerhaft sein Quartier aufzuschlagen.

Von Dezember 1837 bis April 1838 unternahm er dann die Durchquerung der Anden und die anschließende Reise durch Argentinien bis San Luis de la Punta, wobei er auf Grund eines Unfalls überstürzt nach Chile zurückkehren musste. Genau hier setzt der Kurzroman des Argentiniers César Aira, Un episodio en la vida del pintor viajero, ein. ${ }^{3}$ Rugendas befand sich zum damaligen Zeitpunkt in Begleitung eines anderen deutschen Künstlers und Reisemalers, Robert Krause, der mit ihm gemeinsam eine Reihe von Ansichten der Anden entwarf, als Zeichner und Maler aber nicht so sehr im Rampenlicht stand wie der renommierte Maler aus Augsburg.

Von November bis Dezember 1842 folgte die Seereise nach Callao und ab Januar 1843 dann der Aufenthalt in Lima und Peru. Eine Reise nach Arequipa, Tacna und La Paz schloss sich an, schließlich eine erneute Reise über Arequipa

$2 \mathrm{Zu}$ den Beweggründen von Langsdorff vgl. Diener, Pablo: 'Instruction für den Maler welcher nach Brasilien gehen soll’. In: Martius-Staden-Jahrbuch (São Leopoldo) 62 (2018), S. 98-100.

$3 \mathrm{Vgl}$. die deutsche Fassung von Aira, César: Humboldts Schatten. Novelle. Aus dem argentinischen Spanisch von Mathias Strobel. Nachwort von Ottmar Ette. München - Wien: Nagl \& Kimche im Carl Hansere Verlag 2003. 
zurück nach Valparaíso. Im Februar und März 1845 folgte nach einem weiteren kurzen Aufenthalt in Tacna und Valparaíso die Seereise um das Kap Hoorn an den Río de la Plata, wo er sich von März bis Juli 1845 kürzer in Montevideo und deutlich länger in Buenos Aires aufhielt. Von Juli 1845 bis August 1846 erfolgt dann ein letzter Aufenthalt in Rio de Janeiro, wonach er im August 1846 schließlich nach Europa zurückkehrte: Eine lange Reise, ein langer Aufenthalt in den Ländern Lateinamerikas war zu Ende gegangen.

Wie wir sahen, kam Johann Moritz Rugendas auf seiner zweiten, großen Amerikareise im Januar 1843 nach Lima und hielt sich bis September 1844 in der Hauptstadt des Vizekönigreiches von Peru auf. Der Grund, warum ich mich gerade mit dieser Phase seines Künstlerlebens kurz beschäftigen möchte, ist einfach: Flora Tristans Pilgerschaften einer Paria, die in der Alten wie in der Neuen Welt großes Aufsehen erregten, blieben dem Augsburger Maler und Zeichner nicht unbekannt. Seine intensive, in einer Vielzahl von Gemälden und Skizzen deutlich werdende Beschäftigung mit den Tapadas weist viele Merkmale auf, die uns aus unserer bisherigen Auseinandersetzung mit dem Bild der Limanerinnen wohlvertraut sind. Wie sah er diese berühmten und oft beschriebenen Frauen von Lima aus seiner Künstlerischen und männlichen Perspektive?

Viele seiner Darstellungen wirken so, als bildeten Flora Tristans Textpassagen die Ekphrasis eines angekündigten, aber erst noch zu schaffenden Bildes, an dessen Fertigstellung sich der Augsburger gemacht hätte. Auffällig sind die Zweier- und Dreiergruppen verhüllter Frauen, die sich nicht selten als Abwandlung derselben Gestalt in verschiedenen Stellungen - und damit als Bewegungsbilder - deuten lassen. Sie bilden ganze Serien im Werk von Rugendas und lassen sich durchaus in szenische Abfolgen integrieren.

Rugendas war jedoch kein bloßer Augenzeuge, sondern unternahm die ästhetische Bearbeitung einer intermedialen Text-Bild-Beziehung: Ihm stand nicht nur die Realität Modell, sondern auch eine lange literarische Tradition, von der er sehr wohl wusste. Die starke Vertikalität seiner 'verschleierten' Frauen beruht auf einer bewussten Akzentuierung jener Elemente, die über einen langen Zeitraum den literarischen Körper der schönen Limanerin aufgebaut und gebildet hatten: Es handelte sich um die Linie Auge - Taille - Fuß. Bemerkenswert ist, wie häufig Rugendas 'seine' Tapadas mit Männergestalten in Verbindung treten ließ, bei denen es sich kaum um die jeweiligen Ehemänner handeln dürfte. Seine Frauen von Lima bewegen sich in den Zentren des öffentlichen Raumes und genießen ganz offenkundig große Freiheiten.

$\mathrm{Zu}$ dieser starken Akzentuierung einer geschlechtsspezifisch-gesellschaftlichen Dimension kommt nun aber hinzu, dass Rugendas die verhüllten und sich für Augen-Blicke enthüllenden schwarzgekleideten Frauen sehr häufig mit 


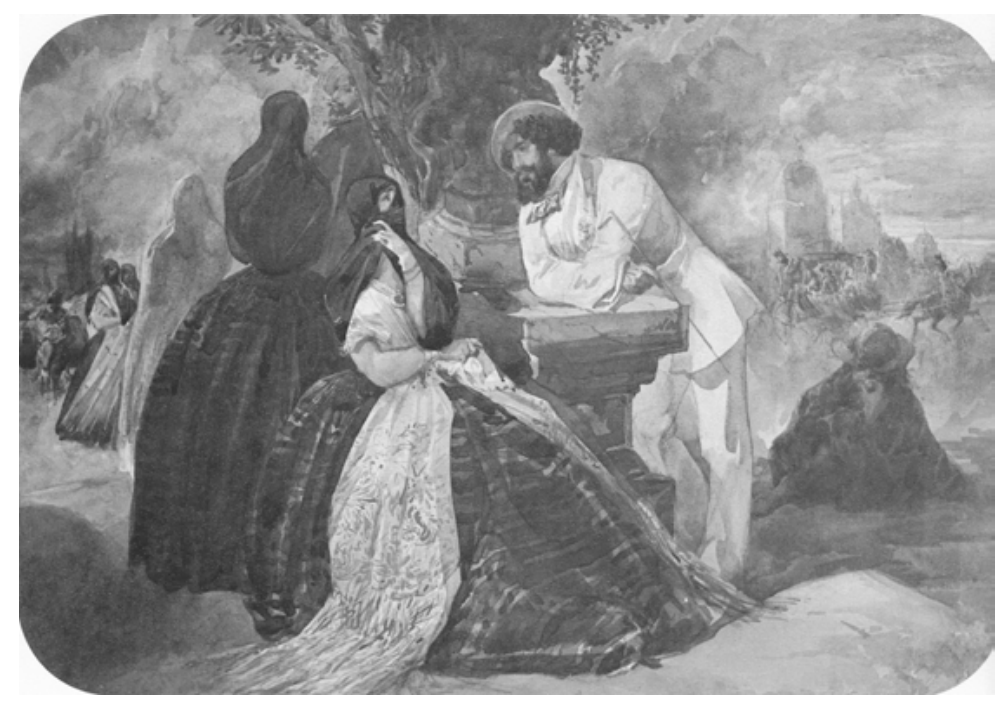

Abb. 84: „Encuentro“ en la alameda nueva, Aquarell von Johann Moritz Rugendas.

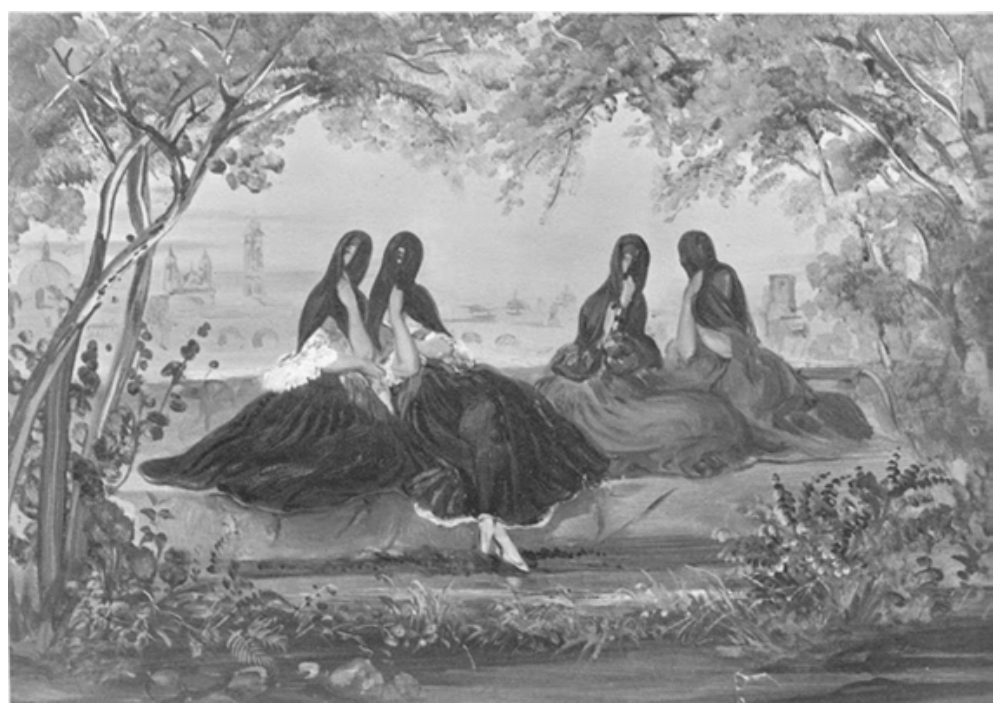

Abb. 85: „Tapadas en la Alameda“, Öl auf Papier von Johann Moritz Rugendas.

einem einzigen enthüllten und den Betrachter des Bildes anblickenden Auge zeigt. Diese Darstellungsweise wirkt ebenso exotisierend wie erotisierend. Da er unter der langen saya oft auch nur einen einzigen kleinen Fuß keck 
hervorschauen lässt, wird der Charakter der Vertikalachse durch die Singularität von Auge, Taille und Fuß eindrucksvoll erhöht. Die Frauen bekommen in seiner Darstellung eine sehr eigene Ausdrucksweise und mobile Statik.

Dabei entwickelt Rugendas, der in seinen künstlerischen Konzeptionen und insbesondere in seinem Verständnis der Landschaftsmalerei in hohem Maße von seinem Förderer Alexander von Humboldt geprägt worden war, eine Darstellungsweise, die den Tapadas nicht selten hochgradig unwahrscheinliche Körperformen und Bewegungen zuschreibt. Die sehr hoch angesetzte und extrem verengte Taille, für deren Beschreibung der Ausdruck 'Wespentaille' (taille de guêpe) nicht ausreicht, der sehr stark verkleinerte und wie bei einer Ballerina hochgestellte Fuß sowie das einzelne, auf die Zuschauer gerichtete Auge (vgl. etwa die während des Aufenthalts in Lima entstandenen Arbeiten 'Die Plaza Mayor von Lima', 'Der Mercado de la Independencia in Lima' oder 'Der Palast von Torre Tagle in Lima') machen deutlich, wie sehr sich bei Rugendas eigenes Sehen und literarische Tradition, Bild und Text, Präsenz und Geschichte des Körpers miteinander verbinden. Rugendas entwickelt das Bild der Tapadas durchaus im Sinne von Flora Tristan weiter: Er gibt diesen Frauen eine eigene Würde, Freiheit und Präsenz.

Wie bei Flora Tristan beherrschen die Frauen zumeist den öffentlichen Raum, den sie mit ihrer schwarzen Silhouette und ihren eigenen wie den auf sie gerichteten Blicken zentrieren. Vom freibleibenden einzelnen Auge einer Tapada geht eine der christlichen Ikonographie entspringende Herrschaft und Macht aus, die oftmals den gesamten Bildraum bündelt und in alle Bereiche des Gemäldes ausstrahlt. Zugleich wird der orientalisierte Frauenkörper unter dem Blick des männlichen europäischen Künstlers erneut zum Objekt eines Begehrens, welches durch das Spiel von Verhüllung und Enthüllung in Bewegung gesetzt wird. Im Gegensatz zu Flora Tristan wird die Tapada bei Rugendas zu einem erotisierten Gegenstand eines Begehrens im männlichen Blick.

Dabei gerät die 'unwahrscheinliche', die Grenzen weiblicher Anatomie sprengende Körperlichkeit mitunter in die Nähe nicht mehr realistischer, sondern unverkennbar symbolhafter Darstellung. Bisweilen aber zeigt sich auch eine Nähe zur Karikatur, die diese Repräsentationen des Weiblichen dank ihrer Übersteigerung unvermutet zum komischen Körper werden lassen - zumindest dann, wenn wir mit Sigmund Freuds Der Witz und seine Beziehungen zum Unbewußten „das Komische [.. .] sich zunächst als ein unbeabsichtigter Fund aus den sozialen Beziehungen der Menschen“ sich entwickeln sehen. Freud betonte die Bindung des Komischen an das Körperliche und an dessen Formen und Bewegungen. Die Überzeichnung stößt in diesen Bereich des Komischen vor, ohne sich darin zu stabilisieren: Das Komische bleibt im Vieldeutigen verankert und entlädt sich nicht. 


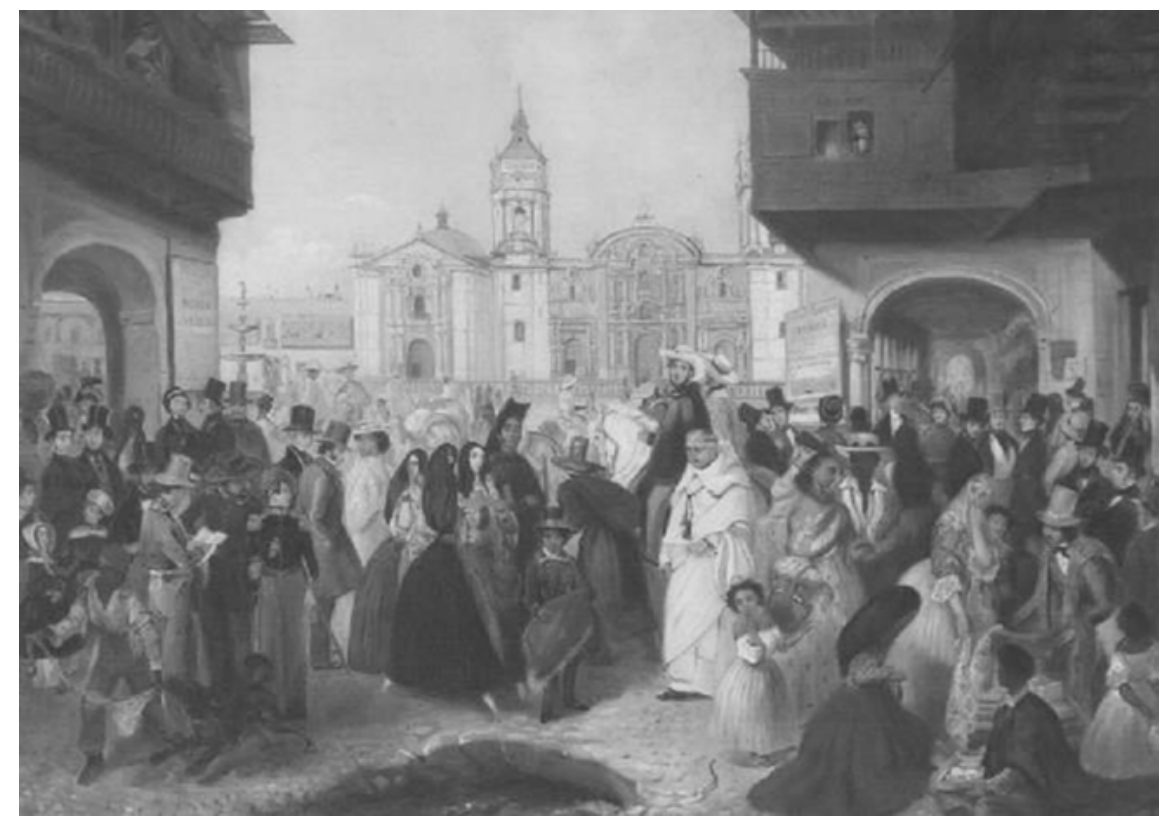

Abb. 86: „La plaza mayor de Lima“, Öl auf Leinwand von Johann Moritz Rugendas, 1843.

So mag verständlich werden, warum die Körper der schönen Limanerinnen bei Rugendas so merk-würdig gebildet sind: Sie stehen in einem so starken Spannungsverhältnis zur literarisch-künstlerischen Tradition, dass die Arbeit am Mythos an diesem Punkt in die Selbstreflexivität einer Körperlichkeit umschlägt, die sich ihrer eigenen literarischen (Vor-) Geschichte nicht mehr entledigen kann. Sie ist ganz einfach Teil ihres Bildes. Im einzelnen Körper verkörpert sich stets eine Geschichte, die den Körper des einzelnen Menschen bei weitem übersteigt.

Die mehr als bemerkenswerte Leistung von Johann Moritz Rugendas dürfte vor allem darin bestehen, in seiner Modellierung des Körpers der schönen $\mathrm{Ta}$ pada all jene Körper sichtbar werden zu lassen, die eine lange Geschichte in ihm versammelt und verdichtet hat. Ästhetisch darstellbar war dies nur, indem er sich den Herausforderungen durch den unwahrscheinlichen und ins Komische umschlagenden Körper stellte und die Körperformen der Frauen ganz neu entwarf. Johann Moritz Rugendas' verschleierte Frauen verhüllen und zeigen zugleich, dass die Geschichte eines Körpers viele Körpergeschichten gespeichert enthält. Doch schließen wir damit unser Kapitel zu den Frauen von und in Lima und den sie betrachtenden Künstlern ab. 